\title{
6 PINPOOLS GmbH - Wenn die Chemie stimmt
}

In jüngerer Vergangenheit hat die Digitalisierung die Normen und Methoden vieler etablierter Unternehmen infrage gestellt. Vor allem digitale Plattformen, die als Intermediäre mehrere Gruppen eines Marktes digital verbinden, haben jüngst an Bedeutung gewonnen und treten mit ihren innovativen Geschäftsmodellen in Wettbewerb zu renommierten Unternehmen. Bei der Düsseldorfer PINPOOLS $\mathrm{GmbH}$ handelt es sich um einen Plattformanbieter, welcher die Beschaffungsprozesse der chemischen Industrie digital abbildet. Die PINPOOLS GmbH bringt Einkäufer und Lieferanten miteinander in Kontakt und garantiert so einen offenen Markt mit transparenten Preisen und Konditionen. Über dieses digitale Netzwerk können Lieferanten neue Kunden/innen gewinnen und mehr Waren verkaufen, wobei Einkäufer/innen gleichzeitig effizient den besten und preisgünstigsten Lieferanten identifizieren können.

Damit junge Unternehmen in dem agilen, schnelllebigen Umfeld der Digitalisierung bestehen und sich gegenüber etablierten Wettbewerbern behaupten können, müssen sie effiziente operationale Arbeitsprozesse implementieren und sich gleichzeitig kontinuierlich weiterentwickeln. Sowohl die Implementierung effizienter Prozesse als auch die ständige Weiterentwicklung der Angebote erfordern konkurrierende Ressourcen und unterschiedliche Mindsets bei den Gründern/innen und ihren Angestellten. Während der Effizienzgedanke in Form von Kosteneinsparungen insbesondere bei der Verbesserung von Arbeitsprozessen vorherrscht, kann die langfristige Wettbewerbsfähigkeit nur gestärkt werden, wenn Unternehmen ein Learning-Mindset haben. Ein solches Learning-Mindset erlaubt es, nach radikalen Produktinnovationen zu suchen, neue Märkte zu beobachten und gewisse Risiken einzugehen.

Dementsprechend verfolgen Unternehmen auf Basis ihrer Mindsets zwei scheinbar gegensätzliche Geschäftsaktivitäten: die Exploitation der bestehenden Kompetenzen, beispielweise bei der Effizienzsteigerung im Tagesgeschäft, und die Exploration von Neuem, beispielsweise in der Produktentwicklung oder der Formulierung der Unternehmensstrategie. Die Ausbalancierung dieser Geschäftsaktivitäten stellt eine große Herausforderung für Gründer/innen junger Unternehmen dar. Im Folgenden wird gezeigt, wie die beiden Gründer Alexander und Heribert Lakemeyer diese Herausforderung mit ihrem Start-up PINPOOLS $\mathrm{GmbH}$ gelöst haben. Der erste Teil beleuchtet die chemische Industrie und die Entwicklung ihres Geschäftsmodells. Dann erfolgt die Analyse, wie die Gründer 
ihre Organisation strukturierten, um alle Geschäftsaktivitäten effizient bearbeiten zu können. Die abschließende Diskussion fasst die Ergebnisse zusammen.

\section{Die chemische Industrie}

Die chemische Industrie befasst sich mit der Herstellung chemischer Erzeugnisse. Sie etablierte sich im Jahr 1850 in Europa und den USA als eigenständiger Wirtschaftszweig und gilt als ein wesentlicher Bestandteil der industriellen Revolution. Dementsprechend kamen die Produkte der Chemieindustrie ursprünglich in der Weiterverarbeitung von Metallen, Motoren, Eisenträgern oder Chemiefasern und Farbstoffen zum Einsatz.

Heute fällt eine genaue Definition von Unternehmen, welche zur Chemieindustrie gezählt werden sollten, schwer. So definiert das Brockhaus-Lexikon die chemische Industrie wie folgt: „[Zur chemischen Industrie gehören] im weiteren Sinne diejenigen Industrien, die sich ausschließlich oder vorwiegend mit der Umwandlung von natürlichen und mit der Herstellung von synthetischen Rohstoffen befassen. Abgrenzungen sind schwierig und nicht einheitlich." ${ }^{1}$ Die Unternehmen der Chemieindustrie wandeln Rohmaterialien wie Öl, natürliches Gas, Luft, Wasser, Metalle oder Mineralien in mehr als 70.000 verschiedene Produkte um. Laut Statistischem Bundesamt gehören die gewerblichen Hersteller der folgenden Produkte zur Chemieindustrie: anorganische Grundstoffe und Chemikalien, organische Grundstoffe und Chemikalien, Düngemittel, Pflanzenbehandlungsmittel und Schädlingsbekämpfungsmittel, Kunststoffe und synthetischer Kautschuk, pharmazeutische Erzeugnisse und sonstige chemische Erzeugnisse (Klebstoffe etc.). Zudem gibt es einige Überschneidungen mit der Kunststoffindustrie, da die meisten Hersteller chemischer Erzeugnisse auch Plastik und Ähnliches produzieren. Dementsprechend stellt die Chemieindustrie viele Produkte her, die andere Industriezweige benötigen, zum Beispiel die Kunststoffindustrie, die Lebensmittelindustrie, die Automobilindustrie, der Maschinenbau, die Glasindustrie und die Baustoffindustrie. Dabei machen Polymer und Plastik etwa 80 Prozent der weltweiten Produktion aus.

Im Jahr 2016 betrug der Umsatz der Chemieindustrie weltweit 3.360 Milliarden Euro. Im Vergleich zum Vorjahreswert von 3.347 Milliarden Euro stieg der Umsatz demnach nur leicht um 0,4 Prozent. Während das Wachstum der weltweiten Chemieindustrie nahezu stagniert, bestehen große Unterschiede in der Wachstumsentwicklung auf regionaler Ebene. Beispielsweise sank der Anteil der EU-Staa-

1 Brockhaus-Enzyklopädie (1987). Mannheim: F.A. Brockhaus GmbH, 19. Auflage, Band 4, S. 447. 
ten am weltweiten Umsatz der Chemieindustrie von 2006 bis 2016 von 28 Prozent auf 15,1 Prozent, während der Umsatz in China im selben Zeitraum von 13,2 Prozent auf 39,6 Prozent drastisch anstieg. Es besteht die Erwartung, dass sich dieser Trend in den kommenden Jahren fortsetzen wird. ${ }^{2}$ In absoluten Zahlen ausgedrückt machte China im Jahr 2016 mit 1.331 Milliarden Euro (39,6 Prozent) den größten Anteil am weltweiten Umsatz aus. Auf Platz zwei und drei folgen die Mitgliedsstatten der NAFTA (USA, Kanada und Mexiko) mit 528 Milliarden Euro Umsatz (15,7 Prozent) und die EU-Mitgliedsstaaten mit 507 Milliarden Euro Umsatz (15,1 Prozent). Die USA produzieren mit 476 Milliarden Euro den größten Anteil der NAFTA-Mitgliedsstaaten, wobei Deutschland mit 145 Milliarden Euro den größten Anteil der EU-Mitgliedsstaaten stellt (28,7 Prozent am EU-Gesamtumsatz von 507 Milliarden Euro). Damit hat sich die EU-Produktion chemischer Erzeugnisse nach der Industriekrise im Jahr 2009 nahezu erholt. So verringerte sich im Laufe der globalen Finanzkrise im Jahr 2008 die Produktion der chemischen Industrie in den EU-Ländern um mehr als 20 Prozent. Ab 2010 konnten sich die Umsatzzahlen jedoch erholen und zeigen inzwischen einen positiven Trend auf dem Niveau der Jahre vor der Finanzkrise. Mit 1,14 Millionen Angestellten im Jahr 2016 befindet sich auch die Mitarbeiteranzahl der chemischen Industrie in Europa auf einem gleichbleibend hohen Niveau.

Für Deutschland ist die Chemieindustrie von besonderer Bedeutung, da sie nach der Automobil- und Maschinenbauindustrie den drittgrößten Wirtschaftszweig im Land darstellt. 6.900 Unternehmen in Deutschland zählen sich zur chemischen Industrie. $\mathrm{Zu}$ den größten deutschen Chemieunternehmen gehören BASF, Linde, LANXESS, EVONIK, Covestro, BAYER und Brenntag. BASF ist der größte Hersteller chemischer Erzeugnisse und BRENNTAG der größte Händler chemischer Produkte weltweit.

Im Jahr 2017 begann die chemische Industrie die vierte Entwicklungsstufe ihrer Geschichte einzuläuten. Im Rahmen der Initiative „Chemie 4.0“ investierten die Unternehmen der Branche vor allem in die Digitalisierung, zirkuläre Wirtschaft und Nachhaltigkeit. So wurden Produktionsanlagen mithilfe der Digitalisierung automatisiert oder virtuelle Realität zur Simulation für Forschung und Produktentwicklung genutzt. ${ }^{3}$ Obwohl die Industrie das große Potenzial der Digitalisierung erkannt hat, fokussiert sie sich bislang hauptsächlich auf Produktinnovationen und vernachlässigt die Vorteile, die eine Digitalisierung der adminis-

2 Vgl. Cefic Facts and Figures of the European chemical Industry (2017), verfügbar unter http://fr.zone-secure.net/13451/451623/\#page=1.

3 Vgl. Verband der Chemischen Industrie e. V. (2017), verfügbar unter https://www.vci.de/ services/publikationen/broschueren-faltblaetter/vci-deloitte-stuide-chemie-4-punkt-0langfassung.jsp. 
trativen Prozesse mit sich bringen würde. Beispielsweise erfolgt eine Geschäftsanbahnung zwischen Einkäufern und Lieferanten meist per Telefon oder E-Mail. Erstaunlicherweise nutzen potenzielle Vertragspartner in 10 bis 20 Prozent der Fälle sogar noch das Faxgerät. Dementsprechend ist der Prozess der Angebotsanfrage ineffizient und der anschließende Preisvergleich zwischen den einzelnen Anbietern sehr umständlich und zeitaufwendig.

\section{Wenn zwei Brüder mit dem Rucksack unterwegs sind}

Die PINPOOLS GmbH wurde im April 2016 von den Brüdern Alexander und Heribert Lakemeyer gegründet. Alexander hat sein Studium zum Diplomkaufmann an der Universität zu Köln im Hauptfach Wirtschaftspsychologie mit dem Nebenfach Unternehmensfinanzierung im Jahr 2012 abgeschlossen. Im Laufe seines Studiums sammelte er Erfahrungen als Praktikant in der Unternehmensberatung, im Controlling und im Vertrieb in Deutschland, Indien und Australien. Zudem kam er in dieser Zeit mit jungen Unternehmensgründern/innen aus seinem Freundes- und Bekanntenkreis in Kontakt, sodass er aus erster Hand die mit einer Unternehmensgründung verbundenen Herausforderungen mitbekam. Die Erzählungen bestärkten ihn in seinem Wunsch, sich auch selbstständig zu machen: „Nach meinen Praktika in verschiedenen Unternehmen war mir klar, dass eine Karriere in einem großen Konzern für mich nicht infrage kommt. Ich wollte schon immer gründen. Aber direkt nach meinem Studium war ich noch nicht so weit. Mir fehlte einfach die weitere Praxiserfahrung und damit auch eine solide Idee. Träumen kann jeder, aber ich wollte schon immer etwas Nachhaltiges mit Substanz aufbauen.“

Die fehlende Praxiserfahrung sammelte Alexander Lakemeyer 2011 bei seinem Berufseinstieg als Assistent der Geschäftsführung eines mittelständischen Kunststoffherstellers. Hier erhielt er als Verantwortlicher für Vertrieb und Controlling einen tiefgehenden Einblick in die Verfahrenstechnik der Kunststoffindustrie. So konnte Alexander Lakemeyer Schwachstellen eines spezifischen Fertigungsprozesses identifizieren und nutzte diese Chance, um sich selbstständig zu machen. Er kündigte seinen Job und gründete 2012 die LAKEMEYER EXTRUSION $\mathrm{GmbH}$. Mit seinem ersten Unternehmen belieferte Alexander und sein Team Kunden/innen aus der Automobilindustrie mit Kunststoffprofilen, die in einem speziellen Inline-Fertigungsverfahren hergestellt wurden.

Letztendlich folgte der Gründung seines ersten Unternehmens die spätere Gründung der PINPOOLS GmbH. Als Geschäftsführer der LAKEMEYER EXTRUSI-

ON GmbH empfand Alexander Lakemeyer den Prozess der Rohstoffbeschaffung in der Plastik- und Chemieindustrie als mühsam und zeitaufwendig. Zum einen 
gibt es viele Großkonzerne und Nischenhersteller mit einer individuellen Produktpalette. Zum anderen variieren die stark volatilen Rohstoffpreise, sodass die potenziellen Einkäufer/innen die Preise für die gewünschte Menge bei den einzelnen Lieferanten/innen anfragen müssen. Außerdem ist die Angebotsnachfrage nicht standardisiert, was dazu führt, dass zahlreiche Telefonate, E-Mails, Faxe und Briefe erforderlich sind. Um diesen Prozess schneller und effizienter abzuwickeln, kam Alexander die Idee, eine digitale, unabhängige Plattform zu initiieren, auf welcher Einkäufer/innen und Lieferanten/innen zielgerichtet verbunden werden können und ein Netzwerk aus Unternehmen der Chemieindustrie entsteht. Die Idee für die PINPOOLS GmbH war geboren. Da das neue Geschäftsmodell zu weit von demjenigen seines ersten Unternehmens entfernt war, entschloss sich Alexander, ein zweites zu gründen. Als potenziellen Mitgründer wollte Alexander unbedingt seinen jüngeren Bruder von seinem Plan überzeugen.

Heribert Lakemeyer studierte International Business an der Universität $\mathrm{Pa}$ derborn mit dem Schwerpunkt Finanzen und Controlling. Nach seinem Bachelorabschluss arbeitete er für ein Jahr in Vollzeit bei einer Unternehmensberatung in Düsseldorf, in der er auch während seines anschließenden Masterstudiums noch in Teilzeit blieb. Heribert konnte sich ebenfalls schon immer vorstellen, ein Unternehmen zu gründen, weswegen er Alexander bereits zuvor beim Aufbau der LAKEMEYER EXTRUSION GmbH unterstützt hatte.

Auf einer gemeinsamen Rucksackreise durch Südamerika im Oktober 2015 erzählte Alexander von seinem Plan, eine digitale B2B-Plattform für die Chemieindustrie zu entwickeln, und fragte Heribert, ob er das Unternehmen als zukünftiger COO mitgründen möchte: „Als frischer Uniabsolvent mit noch relativ begrenzter Praxiserfahrung war ich natürlich etwas überrumpelt. Aber ich habe ohne groß nachzudenken zugesagt. Durch Alexander und meine eigenen Erfahrungen in der Unternehmensberatung wusste ich was für ein Potenzial in der Digitalisierung der Chemieindustrie steckt. Außerdem sind Alexander und ich gemeinsam aufgewachsen und haben schon zusammen für sein erstes Unternehmen gearbeitet. Wir vertrauen uns blind.“

Noch während ihrer Südamerikareise begannen die Brüder Lakemeyer mit der Erarbeitung von Strategie und Konzeption für die PINPOOLS GmbH. Die offizielle Gründung des Unternehmens erfolgte im April 2016 in Düsseldorf. Kurz darauf gingen die beiden Gründer für ein halbes Jahr nach Berlin, um die Start-up-Szene dort kennenzulernen. Heribert erinnert sich: „Wir kamen sehr aus dem analogen Bereich, eine Maschine hinstellen, zu produzieren, an einem Ende der Maschine Rohstoffe hineinkippen, auf der anderen kommt etwas heraus, um das dann zu verkaufen. Sehr analog. Und dann, für ein digitales Business brauchst du was Anderes. Musst Entwickler finden, dich mit anderen Unternehmen austauschen, die schon viel Erfahrung in der digitalen Welt haben." Alexander und Heribert 
knüpften daher Kontakte zu Entwicklern in Berlin und konnten so erste Mitarbeiter/innen einstellen.

Dennoch war es keine Option für die Gründer, in Berlin zu bleiben, da der Standort Düsseldorf für sie wesentlich mehr Vorteile bot, wie Heribert ausführt: „Hier sitzt die Industrie. Also unsere Kunden sitzen hier in der Nähe. Das sind Händler, zum anderen große Konzerne im Rheinland und Ruhrpott. Klar, und in Süddeutschland sitzen ein paar Kunden. Im Frankfurter Raum ein paar und in Ostwestfalen. Aber das ist alles in der Nähe, näher als in Berlin. In Berlin waren wir vorher eher, um die Start-up-Szene kennenzulernen. Da haben wir das gemerkt: Hier sitzt ja niemand. Wir müssen für Kundenbesuche immer quer durch Deutschland fahren. Und vor allem im B2B gehört Persönlichkeit dazu. Du musst die Menschen sehen. Du triffst dich mit denen." Seit Anfang 2017 sind die beiden Gründer mit ihrem Team in einem eigenen Büro im Coworking Space Factory Campus in der Erkrather Straße. Hier arbeiten sie gemeinsam an ihrer Vision: der Digitalisierung der Beschaffungs- und Lieferprozesse in der chemischen Industrie. Bisher können sie auf eine erfolgreiche Gründungsphase mit einem stetigen Mitarbeiter- und Umsatzwachstum und vielen gemeisterten Herausforderungen zurückblicken.

\section{Gegensätze vereinen}

Nach der Gründungsphase war für die Brüder Lakemeyer eine der größten Herausforderungen, wie sie die bereits aufgebauten Kompetenzen ihres Unternehmens effizient nutzen und es flexibel weiterentwickeln konnten. Im organisationalen Kontext spricht man bei der Balance zwischen Effizienz und Flexibilität von „organisationaler Ambidextrie“, also der Fähigkeit, Bestehendes ausnutzen zu können (Exploitation) und gleichzeitig Neues zu erkunden (Exploration). Die Exploitation beinhaltet eine klare Zielorientierung, eine Fokussierung auf Effektivität und die Vermeidung von Risiken und Fehlern. Demgegenüber besteht die Exploration aus dem Experimentieren mit dem Unbekannten, der Ausarbeitung von unkonventionellen Ideen oder das bewusste Eingehen von Risiken. ${ }^{4}$

Studien zeigen, dass Unternehmen, welche diese beiden vermeintlich gegensätzlichen Geschäftsaktivitäten erfolgreich integrieren, eine höhere Innovationsleistung aufweisen als Unternehmen, die sich langfristig auf nur eine der beiden Geschäftsaktivitäten konzentrieren. Außerdem fanden Forscher heraus, dass

4 Vgl. March, J. G. (1991). Exploration and Exploitation in Organizational Learning. Organization Science, 2 (1), S. 71-87. 
Unternehmen mit organisationaler Ambidextrie höhere Wachstumsraten und eine gesteigerte Unternehmensleistung aufweisen. ${ }^{5}$ Die Nutzung aufgebauter Kompetenzen, also die Exploitation, kommt beispielsweise im Tagesgeschäft bei der Implementierung von effizienten operativen Prozessen im Kundenservice, einer funktionierenden Kundenakquise oder der Verbesserung bestehender Produkte zum Tragen. Demgegenüber gehören zur Weiterentwicklung des Unternehmens, also der Exploration, beispielsweise die Formulierung der Strategie, die Entwicklung neuer technischer Innovationen, der Aufbau von strategischen Partnerschaften oder die Expansion in neue Märkte.

Heribert Lakemeyer erinnert sich, wie sie in der Anfangsphase mit der Integration dieser gegensätzlichen Geschäftsaktivitäten umgegangen sind: „Anfangs bestand das Unternehmen ja nur aus Alex, mir und einer Idee. Also haben wir uns zuerst darum gekümmert, dass unsere Idee auch umgesetzt bzw. entwickelt wird. Als wir dann ein funktionierendes Produkt hatten, kam auf einmal ganz viel im Tagesgeschäft dazu, Kundenservice, Kundenakquise usw. Da haben wir beide alles irgendwie gleichzeitig gemacht, aber uns erst mal auf das Operative fokussiert. Manchmal haben wir uns aber auch ganz bewusst ein paar Stunden genommen, um über den Tellerrand zu schauen und unsere Vision nicht aus den Augen zu verlieren."

Die Gründer haben dementsprechend die beiden gegensätzlichen Geschäftsaktivitäten des Tagesgeschäfts und der konzeptionellen Weiterentwicklung ihrer Unternehmung zeitlich getrennt. Während sie sich in der Gründungsphase auf die Umsetzung ihres Geschäftsmodells, also der Exploitation der Organisation, konzentrierten, fokussierten sie sich in der anschließenden Phase mithilfe von Kundenfeedbacks auf die konzeptionelle Ausrichtung und die Weiterentwicklung ihrer Organisation, der Exploration. Dieses Hin- und Herschwanken zwischen Exploitation und Exploration ermöglichte es den beiden Unternehmern, ihre begrenzten Ressourcen in der jeweiligen Gründungsphase bestmöglich einzusetzen. Mit dieser Art der organisationalen Ambidextrie werden die Geschäftsaktivitäten, die auf die Ausbalancierung von Exploitation und Exploration abzielen, zeitlich voneinander getrennt. Dabei wechseln sich meistens lange Phasen der Fokussierung auf die organisationale Effizienz mit kurzen, aber sehr intensiven Phasen der Erforschung und konzeptionellen Weiterentwicklung der Organisation ab. ${ }^{6}$

Ein Nachteil der zeitlichen Trennung der Bearbeitung der verschiedenen Geschäftsaktivitäten besteht darin, dass die Gründer nach ihrer Intuition entschei-

5 Vgl. Raisch, S. und Birkinshaw, J. (2008). Organizational Ambidexterity. Antecedents, Outcomes, and Moderators. Journal of Management, 34 (3), S. 375-409.

6 Vgl. Gupta, A. K., Smith, K. G., und Shalley, C. E. (2006). The Interplay between Exploration and Exploitation. Academy of Management Journal, 49 (4), S. 693-706. 
den müssen, wann sie sich um welche Aufgaben kümmern sollten. Daher fehlt eine klare Aufteilung der Verantwortlichkeiten und eine definierte Organisationsstruktur, die eine zielgerichtete Umsetzung der Vision und Strategie der Unternehmung ermöglicht.

\section{Die Kunst, zu delegieren}

Mit der stetig steigenden Anzahl von Mitarbeiter/innen, dem Umsatzwachstum, der zunehmenden Professionalisierung und der größeren Komplexität ihres Startups stellten sich Alexander und Heribert Lakemeyer die Frage, wie sie es nach der Gründungsphase für eine erfolgreiche Zukunft organisieren und ausrichten müssen. Die Einstellung von Mitarbeitern/innen stellt in der jungen Geschichte eines jeden Start-ups das größte Ereignis im organisationalen Wandel dar. Auch die Brüder standen nun vor der Frage, wie sie die Arbeitsabläufe organisieren und ihre Mitarbeiter/innen führen sollten. Während sie in der Gründungsphase noch alles selbst bearbeitet hatten, konnten sie nun Aufgaben delegieren.

Alexander Lakemeyer erinnert sich, wie sie ihre ersten Delegationsentscheidungen trafen: „Das stellt man sich immer so trivial vor, aber wenn man dann das erste Mal entscheiden muss, welche Aufgaben man an Mitarbeiter abgeben möchte [...], da muss man schon drüber nachdenken. Klar, bei manchen Dingen brauchten wir zusätzliche Kompetenzen wie zum Beispiel bei der Entwicklung des Produkts. Solche Sachen haben wir dann an Experten abgegeben. Im Gegensatz dazu können wir aber auch manche Aufgaben unheimlich gut. Und das geben wir dann natürlich nicht so gerne ab. Aber manchmal hat man einfach nicht genügend Zeit, um alle Aufgaben, die wir gut können und gerne machen, zu behalten. Dann wird auch das delegiert. Und das ist auch kein Problem, weil bei uns ausschließlich Topleute dabei sind!“

Demzufolge entschieden sich Alexander und Heribert Lakemeyer anhand ihrer Kompetenz und Leidenschaft für bestimmte Aufgabenbereiche, was sie delegieren wollten und was nicht. Sie unterschieden vier Fälle:

1. Wenig Kompetenz, wenig Leidenschaft:

Sofern die erforderlichen Ressourcen vorhanden waren, delegierten die Gründer zuerst Aufgaben, für die sie eine geringe Kompetenz und Leidenschaft hatten, an erfahrene Experten/Expertinnen.

2. Hohe Kompetenz, wenig Leidenschaft:

Hierzu zählten die Gründer Aufgaben, welche sie zwar nicht mochten, aber inhaltlich gut bearbeiten konnten. Dennoch könnten diese Aufgaben als lästig und kraftraubend gelten. 
3. Wenig Kompetenz, hohe Leidenschaft:

Das waren Aufgaben, welche die Gründer sehr gerne selbst bearbeiten wollten, obwohl sie nur eine geringe Kompetenz dafür besaßen. Waren diese Aufgaben wichtig genug und ließ es die Zeit und Energie der Gründer zu, konnten sie die nötigen Kompetenzen mithilfe von Trainings über einen längeren Zeitraum aufbauen.

4. Hohe Kompetenz, hohe Leidenschaft:

Hierzu zählten Aufgaben, welche die Gründer sehr gut und gerne bearbeiten wollten. Dazu gehörten solche, welche die Gründer motivieren und üblicherweise den meisten Wert für die Unternehmung schaffen. Diese Aufgaben wollten sie nur unter großem Zeitdruck delegieren.

Da mit der Einstellung ihrer ersten Mitarbeiter/innen die Komplexität der Organisation im Unternehmen stieg, mussten die Gründer eine Lösung für die Bearbeitung von exploitativen und explorativen Aktivitäten finden.

\section{Die Arbeitsaufteilung der Gründer als Vorstufe zur strukturellen Ambidextrie}

Daher entschlossen sich Alexander und Heribert Lakemeyer dazu, eine klare Organisationsstruktur zu etablieren, wie Heribert ausführt: „Formell war Alexander von Anfang an CEO und ich COO. Aber im Prinzip haben wir in der Anfangsphase alles gleichzeitig gemacht [...]. Mädchen für alles, vor allem operativ. Mit unseren ersten Mitarbeitern haben wir dann eine Struktur eingeführt. Allein schon, damit unsere Mitarbeiter wissen, wer von uns für die einzelnen Themen der Ansprechpartner ist. Ich bin als COO für das Operative zuständig, also ich kümmere mich darum, dass das Produkt funktioniert und weiterentwickelt wird, bin Ansprechpartner für unseren Kundenservice usw. Alexander kümmert sich als CEO eher um strategische Themen, wie zum Beispiel den Aufbau von Partnerschaften und den strategischen Vertrieb. Aber auch Alex kümmert sich hier und da um operative Themen, wie zum Beispiel Finanzen und Buchhaltung.“

Alexander Lakemeyers Fokussierung auf Strategie und Weiterentwicklung des Unternehmens und Heribert Lakemeyers Konzentration auf das operative Tagesgeschäft und das Produkt war ein erster Schritt zur strukturellen Ambidextrie. Bei ihr werden exploitative und explorative Aktivitäten strukturell getrennt und erfolgen in unterschiedlichen Geschäftseinheiten. Dadurch beschäftigt sich die Organisation gleichzeitig mit der Verbesserung der Effizienz als auch der Weiterentwicklung des Unternehmens. Ein Vorteil der strukturellen Ambidextrie 
besteht darin, dass Initiativen in Projektteams zielgerichtet organisiert werden können und die individuellen Mitarbeiter/innen ihre Stärken der Exploitation oder Exploration dementsprechend vollständig ausnutzen. Ein Nachteil ist hingegen, dass die Geschäftseinheiten relativ unabhängig voneinander arbeiten und auf eine effiziente Kommunikation miteinander angewiesen sind. Ohne eine funktionierende Kommunikation können nur schwer Synergien zwischen den Geschäftseinheiten genutzt werden. So ist es einerseits beispielsweise denkbar, dass einem Projektteam der Geschäftseinheit, die sich mit der Exploration beschäftigt, gar nicht bekannt ist, welche konkreten Kompetenzen in einem spezifischen Bereich der Organisation bereits vorliegen. Andererseits könnten Mitarbeiter/innen der Exploitation Informationen, welche für die Exploration neuer Geschäftsbereiche wichtig wäre, falsch interpretieren und es versäumen, diese an die mit Exploration beschäftigten Mitarbeiter/innen weiterzuleiten.

Im Fall der Organisation der PINPOOLS GmbH können Schwierigkeiten in der Kommunikation aufgrund der geringen Unternehmensgröße vernachlässigt werden. Außerdem handelt es sich hier per Definitionem nicht um eine strukturelle Ambidextrie, da Exploitation und Exploration nicht in verschiedenen Geschäftseinheiten gebündelt sind, sondern eine klare Aufgabenteilung zwischen den Gründern erfolgt. Dennoch kann die Aufgabenteilung mit Alexander Lakemeyer als CEO, der sich hauptsächlich mit der strategischen Weiterentwicklung und somit der Exploration beschäftigt, und Heribert Lakemeyer, der sich auf ein effizientes Tagesgeschäft und dementsprechend auf die Exploitation vorhandener Kompetenzen fokussiert, als Vorläufer einer struktureller Ambidextrie interpretiert werden.

\section{Gründer als ambidextere Individuen}

Neben der zuvor behandelten temporalen und strukturellen Ambidextrie existiert die kontextabhängige Form der organisationalen Ambidextrie. Die kontextabhängige Ambidextrie zeichnet sich dadurch aus, dass individuelle Mitarbeiter/innen im entsprechenden Unternehmenskontext Aufgaben der organisationalen Effizienz und des Experimentierens mit Neuem eigenständig bearbeiten können und dadurch exploitative und explorative Aktivitäten integrieren. Dabei können sie die Grenzen ihres Jobs überschreiten und Möglichkeiten suchen, um ihre Gedanken und Ergebnisse mit anderen Initiativen zu kombinieren, oder als Multitasker intuitiv zwischen exploitativen und explorativen Aktivitäten wechseln.

Wie in den beiden vorherigen Zitaten deutlich wurde, agieren Alexander und Heribert Lakemeyer in ihrem Unternehmen als ambidextere Individuen. Die Aussagen, dass sie sich zum Beispiel als „Mädchen für alles“ sehen und „irgendwie 
alles gleichzeitig“ machen, lassen darauf schließen, dass sich die beiden Gründer dynamisch zwischen exploitativen und explorativen Aktivitäten hin- und herbewegen. Dabei setzen sie allerdings verschiedene Schwerpunkte: Alexander beschäftigt sich als CEO vorwiegend mit explorativen Aktivitäten, wohingegen Heribert als COO exploitative Aktivitäten im Tagesgeschäft übernimmt. Dennoch ist auch hier nochmals eine Differenzierung notwendig. Obwohl Alexander sich auf die Weiterentwicklung konzentriert, bearbeitet er ebenfalls Aufgaben aus dem Tagesgeschäft, zum Beispiel die Buchhaltung, wohingegen sich Heribert als COO zudem mit explorativen Aufgaben wie beispielsweise der Weiterentwicklung des Produkts beschäftigt.

Demzufolge kombinieren die Gründer der PINPOOLS GmbH temporale, strukturelle und kontextabhängige Charakteristika der organisationalen Ambidextrie. Dabei gelingt es ihnen, eine Organisation aufzubauen, die klare Verantwortlichkeiten für Exploitation und Exploration definiert und infolgedessen eine effiziente Arbeitsteilung ermöglicht. Zusätzlich arbeiten die Brüder sehr eng zusammen und identifizieren so durch die Verknüpfung ihrer unterschiedlichen Verantwortlichkeiten Synergien, die sowohl ein effizienteres Tagesgeschäft als auch die Weiterentwicklung des Unternehmens fördern. Sie agieren als ambidextere Individuen, wobei sie aber durch ihre konkrete inhaltliche Schwerpunktsetzung zugleich klare Verantwortlichkeiten an ihre Mitarbeiter/innen kommunizieren.

\section{Führungsbasierte Ambidextrie}

Gleichzeitig ermutigen Alexander und Heribert Lakemeyer ihre Mitarbeiter/innen, über die Grenzen ihres Aufgabenbereichs hinauszudenken und eigene Vorschläge einzubringen. Heribert erklärt: „Wir wären ja dumm, wenn wir nicht auf unsere Mitarbeiter hören würden. Wir wissen nicht alles besser in unserem Unternehmen und sind froh, dass wir Mitarbeiter haben, die unsere Meinung auch mal hinterfragen. Nur so können wir uns verbessern! Das kreative Potenzial unserer Mitarbeiter ist uns dabei unheimlich wichtig. Aber vor allem am Anfang mussten wir unseren Mitarbeitern zeigen, dass wir uns freuen, wenn sie andere Meinungen vertreten und eigene Vorschläge machen.“

Demnach ermutigen die Brüder ihre Mitarbeiter/innen dazu, eigene Ideen und Verbesserungsvorschläge sowohl bezüglich ihrer funktionalen Aufgaben als auch der internen Organisation einzubringen. Sie sind davon überzeugt, dass traditionelle Top-Down-Organisationsstrukturen Mitarbeiter/innen zu sehr limitieren, da strenge Hierarchien ausschließlich auf eine formalisierte Leistungserbringung abzielen. Stattdessen wollen sie ihre Mitarbeiter/innen durch flache Hierarchien und die Übertragung von Verantwortung dazu motivieren, Wissen 
$\mathrm{zu}$ teilen, neugierig zu sein (auch über ihren jeweiligen Arbeitsbereich hinaus), kreative Verbesserungsvorschläge $\mathrm{zu}$ formulieren und unternehmerisch $\mathrm{zu}$ denken. Dabei versuchen sie vor allem, ihre Mitarbeiter/innen im Operativen auch bei der Weiterentwicklung des Produkts und des Unternehmens einzubeziehen sie möchten also, dass ihre Mitarbeiter/innen explorativ denken. So führen Alexander und Heribert Lakemeyer regelmäßige Mitarbeitergespräche, wo sie unter anderem folgende Fragen stellen: Wenn ihr die Arbeitsprozesse grundsätzlich neu aufsetzen könntet, was würde sich ändern? Wie müsste unser Produkt in ein bis drei Jahren aussehen, damit wir unseren Kunden den bestmöglichen Mehrwert bieten können? Was können wir als Gründer tun, um einen organisationalen Rahmen zu schaffen, damit ihr Bestleistungen bringt? Infolgedessen priorisieren die Brüder eine hohe Transparenz, lassen ihre Mitarbeiter/innen an strategischen Entscheidungen teilhaben und veranstalten regelmäßige Teamevents, um den Zusammenhalt zu stärken.

\section{Kurz und bündig}

Anekdoten zufolge brauche man nur eine gute Idee, um ein erfolgreiches Unternehmen aufzubauen. Dass zwischen der eigentlichen Idee, ihrer Umsetzung und dem folgenden Aufbau zahlreiche vielfältige Herausforderungen gemeistert werden müssen, wird dabei meist außer Acht gelassen. Dabei sind junge Unternehmer/innen nach der initialen Gründungsphase mit vielen verschiedenen Fragestellungen konfrontiert, zum Beispiel: Welche Aufgaben sollten an Mitarbeiter/innen delegiert werden? Für welche Aktivitäten sollten Gründer/innen ihre eigene Zeit und Energie aufwenden? Wie schaffen es Gründer/innen, effiziente Arbeitsabläufe im operativen Tagesgeschäft zu etablieren und gleichzeitig ihre Unternehmung strategisch weiterzuentwickeln? Wie können Unternehmer/innen die Kreativität ihrer Mitarbeiter/innen fördern?

Die Brüder Lakemeyer haben mit ihrem Start-up PINPOOLS GmbH nicht nur gezeigt, wie eine Geschäftsidee konsequent umgesetzt wird, sie konnten zusätzlich eine organisationale Struktur etablieren, die ein effizientes Tagesgeschäft und die Weiterentwicklung des Unternehmens ermöglicht. Die Integration dieser vermeintlich gegensätzlichen Kompetenzen ist vor allem in dem schnelllebigen Umfeld der Digitalisierung und der digitalen Plattformen erforderlich, da Wettbewerber diese Geschäftsmodelle mit wenig Kapital relativ einfach kopieren können. Demzufolge muss die PINPOOLS GmbH ihren Kunden/innen bereits heute einen einzigartigen und verlässlichen Service anbieten. Außerdem muss das Unternehmen sein bisheriges Geschäftsmodell weiterentwickeln und eine Strategie für zu- 
künftige potenzielle Einnahmequellen ausarbeiten wie beispielsweise die Expansion in andere Märkte oder die Entwicklung neuer Produktfeatures.

Alexander und Heribert Lakemeyer haben nach einer turbulenten Gründungsphase einen Weg gefunden, die paradoxe Herausforderung der Integration von Exploitation und Exploration zu meistern. Aufgrund einer effektiven Arbeitsteilung können sie ihre Stärken nutzen: Alexander als CEO beschäftigt sich hauptsächlich mit der strategischen Weiterentwicklung, der Exploration des Unternehmens, während Heribert als COO für die operative Effizienz des Tagesgeschäfts, der Exploitation, zuständig ist. Die enge Zusammenarbeit und das große Vertrauen der beiden Gründer zueinander können so Synergien für den jeweiligen Geschäftsbereich identifizieren.

Zudem geben sie Vertrauen an ihre Mitarbeiter/innen weiter, indem sie sie ermutigen, eigene Ideen einzubringen und die Vorgehensweise der beiden Gründer regelmäßig zu hinterfragen. Demzufolge schafft es das Düsseldorfer Start-up PINPOOLS GmbH mit seiner Organisationsstruktur, die individuellen Stärken von allen Beteiligten zu fördern. Damit ist es bestens für das weitere Unternehmenswachstum aufgestellt. 


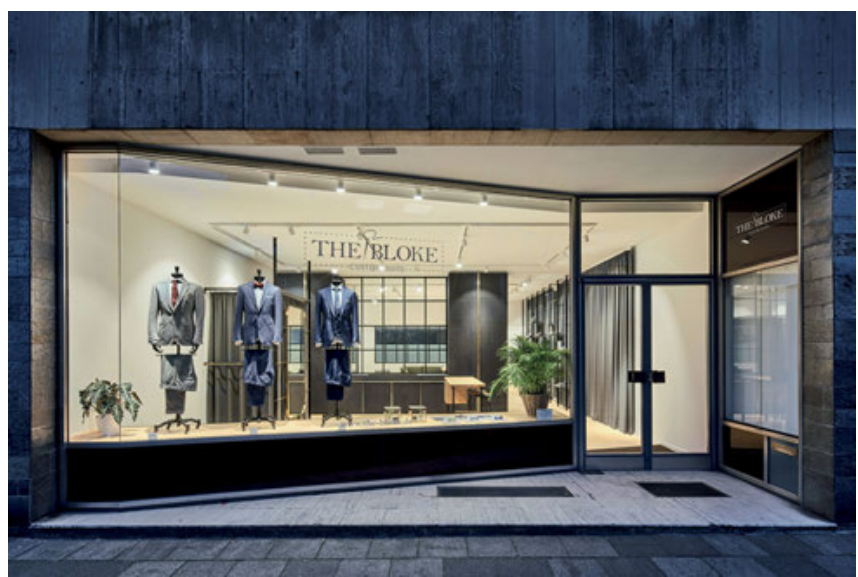

The Bloke Ladenlokal (Bildnachweis: Annika Feuss Fotografie)

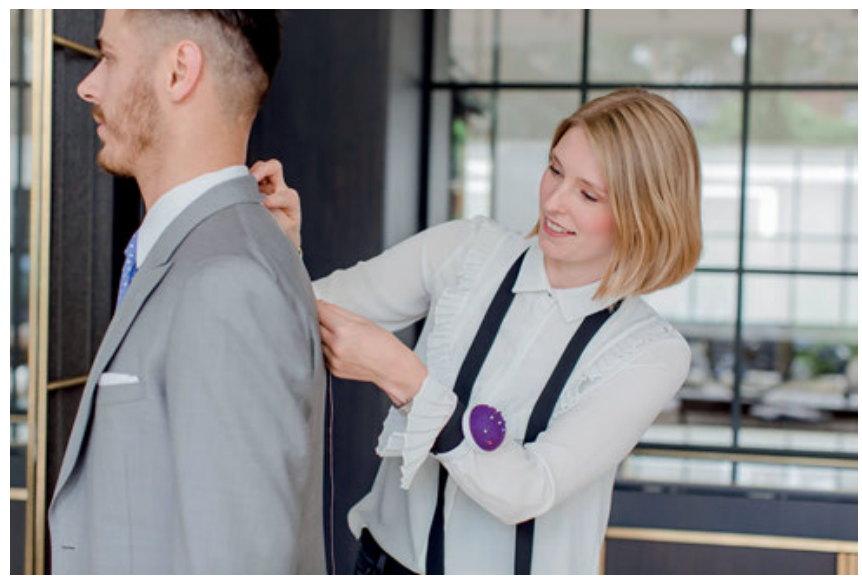

Die Gründerin von The Bloke - Anna Ullrich-Cattien (Bildnachweis: Hanna Witte) 